doi: http://dx.doi.org/10.7124/bc.0009C6

\section{Nuclear organization affects B-cell lymphomagenesis}

Diego Germini, Fatimata Bintou Sall, Vassily Khammad and Yegor Vassetzky

UMR8126, Université Paris Sud - Paris Saclay, CNRS, Institut Gustave Roussy, 94805 Villejuif, France; LIA 1066, French-Russian Joint Cancer Research Laboratory, 94805 Villejuif, France, 119334

Moscow, Russia

vassetzky@gmail.com

Recently we discovered a novel mechanism explaining how B-cell lymphomas might be induced during HIV infections. HIV-positive subjects have an increased risk to develop specific lymphoma subtypes including Burkitt lymphoma (BL). We recently found that viral transactivator of transcription (Tat) protein, which is released by infected cells into the blood stream, could remodel the B-cell nucleus bringing together the potential translocation partners, the MYC loci at the chromosome 8 and the IGH loci at the chromosome 14, thus increasing the probability of the $t(8 ; 14)$ translocation characteristic of BL. Tat induces the mobility of the MYC locus in the nucleus via induction of DSB in the vicinity of the MYC gene and its further repair by NHEJ. In order to study the mechanism of the DSB/NHEJinduced locus relocalization, we have created and characterized the lymphoblastoid RPMI8866 cell line inducibly expressing CRISPR/Cas 9 and gRNA targeting the upstream region of the MYC IGH genes. Upon induction, This leads to relocalization of the MYC locus towards the center of the nucleus and the IGH CRISPR/Cas9 generates DBDs in the target loci as well as $\mathrm{t}(8 ; 14)$ transloca- tions. Factors that increase the proximity between the MYC and IGH loci also increase the $t(8 ; 14)$ frequency and inversely, drugs that inhibit proximity also inhibit the translocation frequency. Therefore, we provide here the first experimental proof that spatial proximity indeed increases the probability of chromosomal translocations.

doi: http://dx.doi.org/10.7124/bc.0009C7

\section{Dysregulation of splicing factors in B-cell acute lymphoblastic leukemia}

\section{Andrei Thomas-Tikhonenko}

Division of Cancer Pathobiology of the Children's Hospital of Philadelphia and Perelman School of Medicine at the University of Pennsylvania, Philadelphia, PA 19104, USA

Aberrant splicing is a hallmark of leukemias with mutations in splicing factor (SF)-encoding genes. We investigated its prevalence in pediatric B-cell acute lymphoblastic leukemias (B-ALL), where SFs are not mutated. By comparing them to normal pro-B cells, we found thousands of aberrant local splice variations (LSVs) per sample, with 279 LSVs in 241 genes present in every comparison. These genes were enriched in RNA processing pathways and encoded $\sim 100$ SFs, e.g. hnRNPA1. hnRNPA1 3'UTR was most pervasively mis-spliced, yielding the transcript subject to nonsense-mediated decay. To mimic this event, we knocked it down in B-lymphoblastoid cells and identified 213 hnRNPA1-dependent aberrant exon usage events comprising the hnRNPA1 splicing sig- 
nature in pediatric leukemia. Some of its elements were LSVs in DICER1 and NT5C2, known cancer genes. More broadly, the twenty most common B-ALL drivers (including NT5C2) showed higher prevalence of aberrant splicing than of somatic mutations. Thus, posttranscriptional deregulation of SF can drive widespread changes in B-ALL splicing and likely contributes to disease pathogenesis.

doi: http://dx.doi.org/10.7124/bc.0009C8

\section{Cancer-testis antigen MAGEA protein expression in extracellular vesicles released by cells}

\author{
Anneli Kuldkepp, Olavi Reinsalu, Magda \\ Karakai, Eve Toomsoo, and Reet Kurg \\ Institute of Technology, University of Tartu, Tartu, \\ Estonia \\ reet.kurg@ut.ee
}

To study the possible role of cancer testis antigens MAGEA proteins in cellular processes triggering normal cells to cancerous. The specific aim is to characterize MAGEA-positive extracellular vesicles (EVs). MAGEAs represent a unique class of tumor antigens which are expressed in a wide variety of malignant tumors, while their expression in healthy normal tissues is restricted to germ cells of testis, fetal ovary and placenta. Their restricted expression and immunogenicity make them ideal targets for immunotherapy in human cancers. MAGEA expression is observed mainly in cancers that have acquired malignant phe- notypes, invasiveness or metastasis, and the expression of MAGEA family proteins has been linked to a poor prognosis in cancer patients. MAGEA proteins have shown to interact ubiquitin ligases and regulate proteasome degradation of proteins, regulate the stability of p53 and interfere with the apoptotic pathway of the cell. Methods: MAGEA-positive EVs are isolated from cell culture media using differential ultracentrifugation and characterized by various biochemical and cellular methods, including western blot analysis, flow cytometry and confocal microscopy. Results: We show that MAGEA proteins are incorporated into extracellular vesicles of different sizes ranging from $150 \mathrm{~nm}$ to $2 \mu \mathrm{m}$ as well as to retrovirus Gag-induced virus-like particles (VLPs). MAGEA proteins, locating both in the nucleus and cytoplasm of the cell, are directed to the surface of EVs released by cells and furthermore, induce the shedding of cells. MAGEA proteins can also be used to guide recombinant proteins, e.g. EGFP and Cherry, onto the surface of EVs allowing to follow the behavior of EVs in real time. In addition, we show that MAGEA10 expression in mouse fibroblasts induces abnormal phenotypes, where the cell has multiple small nuclei. The mechanism of this phenomena and its biological significance is still not known and needs further investigations. Conclusions: We show that MAGEA proteins are incorporated into EVs and are exposed on the surface of EVs. This process is induced, at least to some extent, by the expression of MAGEA proteins itself and may have a role in formation of cancer metastasis. 